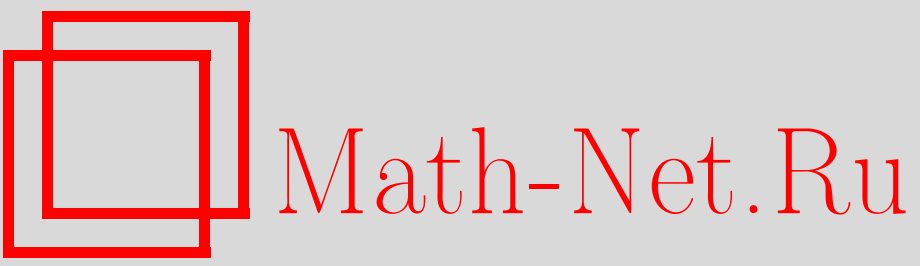

М. И. Штогрин, Примитивные полициклы: критерий, УМН, 1999, том 54, выпуск 6, 177-178

DOI: https://doi.org/10.4213/rm245

Использование Общероссийского математического портала Math-Net.Ru подразумевает, что вы прочитали и согласны с пользовательским соглашением

http://www.mathnet.ru/rus/agreement

Параметры загрузки:

IP : 3.82 .47 .9

26 апреля 2023 г., 06:02:55 


\title{
ПРИМИТИВНЫЕ ПОЛИЦИКЛЫ: КРИТЕРИЙ
}

\author{
М.И. Штогрин
}

В работе [1] для плоского ${ }^{1}$ граффа $G$ определено понятие примитивного поли- $r$-цикла $\Pi(G)$ как всей конечной части плоской карты, удовлетворяющей трем условиям (1)-(3). Там же была доказана теорема 1: если граф $G$ допускает реализацию полицикла $\Pi(G)$ как конечной части плоской карты с условиями (1)-(3), см. [1], то все такие реализации изоморфны.

Эту теорему естественно назвать теоремой единственности, так как любой планарный граф $G$ с точностью до изоморфизма может допускать только одну реализацию соответствующего ему поли- $r$-цикла $\Pi(G)$. Более точно, реберный остов тетраэдра $\mathbf{K}\left(3^{3}\right)$, куба $\mathbf{K}\left(4^{3}\right)$, додекаэдра $\mathbf{K}\left(5^{3}\right)$ имеет соответственно $4,6,12$ разных реализаций, однако все они изоморфны. Устройство поли- $r$-цикла $\Pi(G)$ будем представлять посредством комбинаторно изоморфного ему абстрактного полиэдра $\mathbf{P}(G)$, составленного из правильных евклидовых $r$-угольников. Тогда $\Pi\left(3^{3}\right) \sim$ $\mathbf{P}\left(3^{3}\right)=\mathbf{K}\left(3^{3}\right)-\mathbf{f}, \mathbf{\Pi}\left(4^{3}\right) \sim \mathbf{P}\left(4^{3}\right)=\mathbf{K}\left(4^{3}\right)-\mathbf{f}, \mathbf{\Pi}\left(5^{3}\right) \sim \mathbf{P}\left(5^{3}\right)=\mathbf{K}\left(5^{3}\right)-\mathbf{f}$, где через $\mathbf{f}$ обозначена грань многогранника. Из тетраэдра, куба, додекаэдра выброшена одна грань, все равно какая (так как иначе полиэдр был бы гомеоморфен сфере $\mathbb{S}^{2}$ и не вкладывался бы в евклидову плоскость $\mathbb{R}^{2}$ ). Именно по этой причине указанное выше число реализаций совпало с числом граней многогранника. У любого другого допустимого графа, отличного от реберного остова тетраэдра, куба и додекаэдра, реализация единственна.

Ниже для данного планарного графа $G$ решается вопрос о существовании поли- $r$-цикла $\Pi(G)$, исходя из свойств самого граффа $G$. А именно, имеет место следующий

КРИтЕРиЙ. Отличный от реберного остова тетраэдра, куба, додекаэдра планарный граф $G$ допускает на плоскости реализацию примитивного поли-г-цикла $\Pi(G)$ как всей конечной части плоской карты с условиями (1)-(3) из [1] тогда и только тогда, когда

(а) каждое ребро принадлежит одному или двум простым циклам кратчайшей ${ }^{2}$ длины $r$

(b) те ребра, каждое из которых принадлежит одному $r$-циклу, составляют простой иикл;

(c) пересечение любъх двух г-циклов графа либо есть только одно ребро, либо пусто;

(d) число всех вместе взятых вершин и $r$-циклов графа превышает число ребер ровно на 1.

Каждое ребро из реберного остова тетраэдра, куба, додекаэдра принадлежит двум циклам длины $3,4,5$ соответственно. Если на каждый $r$-цикл натянута грань, получится замкнутый многогранник, гомеоморфный сфере. После выбрасывания одной грани получится полиэдр с краем, изоморфный полициклу. Его край есть край выкинутой грани, т.е. $r$-цикл, $r=3,4,5$.

В случае любого другого допустимого граффа $G$ в силу $[1 ;$ следствие из основной леммы] любой $r$-цикл поли- $r$-цикла $\Pi(G)$ ограничивает лишь внутреннюю грань плоского графа $G$.

Необходимость. Рассмотрим полицикл, удовлетворяющий условиям (1)-(3). В силу условия (2) все грани поли- $r$-цикла $\Pi(G)$ (т.е. внутренние грани плоского граффа $G$ ) суть диски, ограниченные простыми циклами длины $r$. Любое внутреннее ребро поли- $r$-цикла $\Pi(G)$ принадлежит двум граням, являясь их пересечением. Любое граничное ребро поли- $r$-цикла $\Pi(G)$ принадлежит только одной грани. А так как в поли- $r$-цикле $\Pi(G)$, отличном от $\Pi\left(3^{3}\right)$, $\Pi\left(4^{3}\right), \Pi\left(5^{3}\right)$, любой $r$-цикл ограничивает грань (см. [1] и рассуждения выше), где $r=g(G)$, то выполнено условие (а). По той же причине условия (1) и (3) можно переформулировать в виде (b) и (c). Кроме того, для полицикла имеет место формула Эйлера $\boldsymbol{\theta}-p+2=1$, в которой не хватает всего лишь одной грани, которую следует натянуть на край поли- $r$-цикла $\Pi(G)$ для получения сферы. Поэтому выполнено и последнее условие (d).

Работа выполнена при поддержке Российского фонда фундаментальных исследований (грант № 98-01-00251).

${ }^{1}$ Употребляемая здесь терминология из теории графов взята из книги [2].

${ }^{2}$ Кратчайший цикл в графе $G$ называют обхватом граффа и обозначают через $g(G)$; значит, $r=g(G)$. 
Достаточность. Рассмотрим планарный граф, удовлетворяющий условиям (a)-(d). Натянем грани на все $r$-циклы, а также на тот простой цикл $z$, который составлен из всех ребер графа $G$, каждое из которых принадлежит лишь одному $r$-циклу, см. условие (b). В силу условия (a) степень вершины граф̆а не меньше 2. В силу условия (c) в вершине граффа сходится не более трех $r$-циклов. Три $r$-цикла составляют циклическую последовательность вокруг вершины. Если число $r$-циклов меньше трех, то они входят в одну циклическую последовательность вместе с циклом $z$ из условия (b). Поэтому получается многообразие. Выкинем одну излишнюю грань, натянутую на цикл $z$. Тогда в силу (d) останется в точности диск, составленный из одних $r$-угольников, удовлетворяющий условиям (1)-(3).

ЗАМЕЧАНИЕ. Для реального получения поли- $r$-цикла из данного графа при выполнении условий критерия следует натянуть диски на все $r$-циклы граффа. Полученный поли- $r$-цикл при желании можно вложить в плоскость, причем только единственным способом, см. [1].

ПримечАниЕ. Натянув грани на все $r$-циклы графа, получим абстрактный двумерный полиэдр, судя по свойствам которого всем выполняющимся условиям критерия можно придать следуюший смысловой оттенок: (a) - неразветвленность в ребрах, (b) - отсутствие дыр, (c) - клеточный комплекс с примитивными схождениями в вершинах, (d) - односвязность.

ПримеР 1. Для простой цепи, состоящей из 3 ребер додекаэдра, возможно одно из двух: либо эта цепь не принадлежит одной грани додекаэдра, и тогда она является частью простого цикла, длина которого больше 6, так как сама эта цепь является единственной кратчайшей между своими концами, либо эта цеп принадлежит одной грани, и тогда она является частью кратчайшего простого цикла, длина которого равна 5 , совпадающего с краем этой грани. Следовательно, любой кратчайший простой реберный цикл додекаэдра ограничивает грань. Это свойство по наследству сохраняет половина поверхности додекаэдра: корзина с дном и пятью боковыми гранями. Реберный остов корзины удовлетворяет условиям (a)-(d). Проекция корзины на плоскость дна представляет собою поли-5-цикл. Реберный остов корзины как планарный граф̆ имеет шесть других укладок на плоскости, однако для них нарушено условие (2): среди внутренних граней кроме пяти 5-угольников имеется один 10-угольник. Из 7 различных укладок только одной соответствует полицикл. Это согласуется с теоремой единственности [1], которая не отрицает наличия других укладок планарного граффа на плоскости, а лишь утверждает, что среди всех неизоморфных укладок может находиться только один поли- $r$-цикл. (Это своеобразный аналог известной теоремы $[2 ;$ теорема 11.5$]$.)

ПРИмеР 2. Из трех правильных 8-угольников можно склеить правильную треугольную призму. Возьмем две такие призмы с одним общим 8-угольником, обладающие скрещивающимися перпендикулярными осями, расположив их по разные стороны от плоскости общего 8-угольника. Получим своеобразный граненый перстень - полиэдр, составленный из пяти правильных 8-угольников, обладающий зеркально-поворотной осью четвертого порядка.

Реберный остов этого полиэдра является планарным графом, который имеет восемь различных укладок на плоскости, в каждой укладке имеется по семь внутренних граней. Существует четыре изоморфные укладки с четырьмя 8-угольными и тремя 9-угольными гранями и четыре изоморфные укладки с тремя 8-угольными и четырьмя 9-угольными гранями. Ни одна укладка данного планарного графа на плоскости не удовлетворяет условию (2).

Рассматриваемый полиэдр состоит из пяти правильных 8-уголных граней, его реберный остов удовлетворяет условиям (a)-(c), но не удовлетворяет условию (d) предъявленного выше критерия. Учитывая примечание, данный полиэдр не является односвязным примитивным поли-8-циклом. Если угодно, его можно назвать неодносвязным примитивным поли-8-циклом. Этот полиэдр представляет собою сферу с одной дырой и одной ручкой, и наличие ручки служит реальной помехой для существования вложения этого полиэдра в плоскость $\mathbb{R}^{2}$. Поэтому не существует комбинаторного отображения рассматриваемого полиэдра в $\left(8^{3}\right)$.

\section{СПИСОК ЛИТЕРАТУРЫ}

[1] Деза М., Штогрин М.И. // УМН. 1999. Т. 54. №6. С. 159-160. [2] Харари Ф. Теория графов. М.: Мир, 1973.

LIENS, Ecole Normale Supérieure and CNRS, Paris; Математический институт им. В. А. Стеклова РАН 